\title{
Ursolic acid inhibits growth and induces apoptosis in gemcitabine-resistant human pancreatic cancer via the JNK and PI3K/Akt/NF-kB pathways
}

\author{
JINGJIE LI, XIAOYAN LIANG and XING YANG \\ Center of Reproductive Medicine, The Sixth Affiliated Hospital, Sun Yat-sen University, Guangzhou, P.R. China
}

Received March 16, 2012; Accepted April 30, 2012

DOI: $10.3892 /$ or.2012.1827

\begin{abstract}
Pancreatic cancer is one of the most deadly carcinomas worldwide. Although gemcitabine as the standard chemotherapy agent has been proven to be effective, the response rate remains at $5.4 \%$ and the 5-year survival rate is extremely poor. Ursolic acid (UA) is a small molecule compound extracted from Chinese herbs as well as edible vegetables and a well-known anti-inflammatory and immunosuppressive agent. Here, we show that UA has potential to be developed into an anti-neoplastic agent against gemcitabine-resistant pancreatic cancer and to explore its molecular mechanism of action. In vitro, we used three different malignancy grades of pancreatic resistant cancer cell lines including MIA PaCa-2, PANC-1 and Capan-1 to assess the antitumor effect of UA. We found that UA inhibited growth and induced apoptosis in a dose-dependent manner in all of the three pancreatic cancer cell lines. Both extrinsic and intrinsic pathways were found to be involved in apoptotic cascade. The potential signaling pathways are concerned with inactivation of the PI3K/Akt/ $\mathrm{NF}-\kappa \mathrm{B}$ pathway and activation of the c-Jun-terminal kinase (JNK) pathway. The JNK inhibitor SP600125 partly abrogated the caspase- 9 activation caused by UA. The Akt inhibitor LY294002 did not mimic the effect of UA on caspase-8 and -9 , but inhibited the viability of MIA PaCa- 2 cells to some extent. Equally, UA also overcame the chemoresistance in the chemoresistant endometrial and ovarian carcinoma cell lines (HEC-1A and OVCAR-3). Moreover, UA caused cytotoxicity to a nude mouse xenograft model in vivo. Therefore, our present data suggest that UA can act as a novel and potent therapeutic agent in gemcitabine-resistant pancreatic cancer and even as a promising candidate in other chemoresistant cancers.
\end{abstract}

Correspondence to: Dr Xiaoyan Liang, Center of Reproductive Medicine, The Sixth Affiliated Hospital, Sun Yat-sen University, No. 17 Shou-gou-ling Road, Guangzhou 510080, P.R. China E-mail:1xyzy@263.net

Key words: ursolic acid, apoptosis, gemcitabine-resistant pancreatic cancer, JNK, PI3K/Akt/NF- $\mathrm{KB}$

\section{Introduction}

Pancreatic cancer, characterized by aggressive malignancy and high resistance to chemotherapy agents, is considered as one of the most lethal tumors with a 5-year mortality of $97-98 \%(1,2)$. Surgery is the only curative treatment for pancreatic cancer. However, $<20 \%$ patients have resectable tumor at the time of diagnosis due to the poor diagnosis (3). Except for the surgical therapy, both of chemotherapy and radiation regimens hardly ameliorate the outcome of pancreatic cancer patients, which causes $\sim 90 \%$ of cases death within 1 year after diagnosis (4). Since the end of 20th century, gemcitabine, a deoxycitidine analogue, was confirmed to improve the objective response rate in patients with advanced pancreatic carcinoma, and has been applied as a first-line chemotherapy agent $(5,6)$. However, only $5.4 \%$ of all patients are sensitive to the gemcitabine-based chemotherapy $(7,8)$. Thus, novel chemotherapeutic drugs and candidate compounds are urgently needed to improve the outcome of patients with the lethal malignancy.

Ursolic acid (UA) is a pentacyclic triterpenoid compound extracted and purified from many types of medicinal plants, herbs, and edible vegetables (9). UA contains a variety of pharmacological activities. The anti-oxidative and anti-inflammatory actions of UA are widely applied to hepatoprotection, neuroprotection, cardiovascular protection and so on (10-14). Besides these effects, UA has been proved to possess multiple anti-tumor activities including inhibiting tumorigenesis and progression (9). UA causes cytotoxicity to diverse malignancies such as fibrosarcoma, prostate cancer, hepatocellular carcinoma, colorectal cancer, melanoma, and breast cancer (15-20). However, the cytotoxic role of UA in gemcitabine-resistant human pancreatic cancer and its potential molecular mechanisms are still unclear.

In this study, we investigated the effect of UA on growth restriction and apoptosis in human pancreatic resistant cancer in vitro and in vivo. Additionally, we explored possible mechanisms of action, such as PI3K/Akt/NF- $\kappa \mathrm{B}$ and c-Jun N-terminal kinase (JNK) pathways.

\section{Materials and methods}

Cell culture and reagents. The human pancreatic resistant cancer cell lines MIA PaCa-2 and PANC-1 were kindly provided by Shanghai Institute of Materia Medica. The human pancreatic 
resistant cancer cell line Capan-1 was kindly provided by Department of Pharmacology, Sun Yat-Sen University. The paclitaxel-resistant endometrial cell line HEC-1A was purchased from Cell Bank of Chinese Academy of Sciences. The platinumresistant ovarian cancer cell line OVCAR-3 was purchased from Experimental Animal Center of Sun Yat-Sen University. The cells were routinely cultured in DMEM (Invitrogen, Grand Island, NY) supplemented with $10 \%$ fetal bovine serum (FBS) (Invitrogen), $100 \mathrm{U} / \mathrm{ml}$ penicillin, and $100 \mathrm{mg} / \mathrm{ml}$ streptomycin in $5 \% \mathrm{CO}_{2}$ at $37^{\circ} \mathrm{C}$. Ursolic acid (UA) (National Institutes for Food and Drug Control, China) was dissolved in DMSO (SigmaAldrich, St. Louis, MO) as a $100 \mathrm{mM}$ stock solution and stored at $-20^{\circ} \mathrm{C}$. Further dilution was done in serum-free cell culture medium. PI3K inhibitor LY294002 (Cell Signaling Technology, Beverly, MA) was dissolved in DMSO (Sigma) as a $50 \mathrm{mM}$ stock solution and stored at $-20^{\circ} \mathrm{C}$. Similarly, JNK inhibitor SP600125 (Sigma) was dissolved and stored as a $10 \mathrm{mM}$ stock solution by DMSO at $-20^{\circ} \mathrm{C}$.

Cell viability and proliferation. Cells were seeded into 96-well plates at a density of $2 \times 10^{3} /$ well and allowed to attach overnight before being treated by different concentrations of UA for $24 \mathrm{~h}$. Then cell viability and proliferation were evaluated. Cell viability was tested by the 3-(4,5-dimethylthiaziazol-2-yl)-2, 5-diphenyl tetrazolium bromide (MTT, Sigma) assay as described in the literature (21). Detection of cell proliferation was through the colorimetric 5'-bromo-2'-deoxy-uridine (BrdU) incorporation assay (Roche Diagnostic Corp., Indianapolis, IN, USA) according to the instruction of the manufacturer. Briefly, cells were labeled by addition of $\mathrm{BrdU}$ for $4 \mathrm{~h}$ in $5 \% \mathrm{CO}_{2}$ at $37^{\circ} \mathrm{C}$. Then, FixDenat solution was used for cells fixation. The anti-BrdU-POD antibody was added to locate the BrdU label in the DNA. After the incubation of the peroxidase substrate, the immune complex was detected by a multi-well spectrophotometer reader using an iMark ${ }^{\mathrm{TM}}$ Microplate Reader (Bio-Rad, Richmond, CA).

Hoechst 33258 staining. The three types of cells at the density of $2 \times 10^{6} /$ well in a 6 -well plate were fixed by $4 \%$ paraform for $10 \mathrm{~min}$. Then Hoechst 33258 (Sigma) at $5 \mu \mathrm{g} / \mathrm{ml}$ was added to the cells for $15 \mathrm{~min}$ at room temperature in the dark. The nuclei morphology was observed by fluorescence microscopy (Olympus, Melvie, NY) with a 340-nm excitation filter.

Caspase-3/7, - 8 and -9 activity measurements. Caspase-3/7, -8 and -9 activity were assessed by Caspase-Glo luminescentbased assays (Promega, Madison, WI). Accordance with the instructions, cells $\left(1 \times 10^{4} /\right.$ well) were seeded into a 96-well plate overnight to ensure adherence. After various concentrations of UA treatment for $24 \mathrm{~h}$ in a $5 \% \mathrm{CO}_{2}$-humidified atmosphere at $37^{\circ} \mathrm{C}, 100 \mu \mathrm{l}$ of $3 / 7,-8$ and -9 Caspase-Glo reagents were added to each well, respectively. Mixed and incubated for $1 \mathrm{~h}$, the mixtures were transferred to a fluorescence microtiter plate and quantified fluorescence intensity by a luminometer. The corresponding OD values from MTT assay described above were considered as the viable cell number to normalize the fluorescence intensity.

Cytochrome C ELISA assay. Cytoplasmic cytochrome C levels were determined by a cytochrome C ELISA kit (R\&D Systems, Minneapolis, MN). The cytoplasmic protein was collected by a nuclear and cytoplasmic extraction reagents (Pierce, Rockford,
IL) from adherent cells (2x10 $/$ well) in a 6-well plate after UA incubation for $24 \mathrm{~h}$. The BCA protein assay kit (Pierce) was used to normalize the cytoplasmic protein. The protein was stored in $-80^{\circ} \mathrm{C}$ until it was analyzed by cytochrome C ELISA kit according to the manufacturer's instructions.

Western blotting and immunofluorescent analysis. Western blotting was performed as described previously (22). The following antibodies were used: antibodies against PI3K, Akt, phospho-Akt, GSK-3 $\beta$, phospho-GSK-3 $\beta$, NF- $\kappa$ B phospho-p65, JNK, phospho-SAPK/JNK, phospho-MDM2, IкB (1:1000, Cell Signaling Technology); NF-кB p65, phospho-ІкB (1:1000, Santa Cruz Biotechnology, Santa Cruz, CA), and actin (1:1000, Thermo Scientific IHC, Fremont, CA).

The immunofluorescent analysis was performed as previous protocol (23). After UA treatments, the MIA PaCa-2 cells were fixed in $4 \%$ paraformaldehyde for $30 \mathrm{~min}$ and perforated by PBS containing $0.1 \%$ Triton X-100. The cells were blocked with $5 \%$ bovine serum albumin (BSA) for $1 \mathrm{~h}$ at $37^{\circ} \mathrm{C}$ and incubated the NF- $\kappa$ B p 65 antibody $(1: 200)$ at $4^{\circ} \mathrm{C}$ overnight. After washing by PBS, Cy3-conjugated goat anti-rabbit $\operatorname{IgG}$ (1:50, Proteintech, Chicago, IL) was used as the secondary antibody for $1 \mathrm{~h}$ at $37^{\circ} \mathrm{C}$. Nuclear staining was achieved by incubating the cells in DAPI for $10 \mathrm{~min}$. The fluorescence was photographed with fluorescence microscopy (Olympus).

Mouse xenograft model and immunohistochemical analysis (IHC). To establish a subcutaneous xenograft model, MIA PaCa-2 cells $\left(5 \times 10^{6}\right)$ were inoculated subcutaneously on the flanks of 6-week-old nude mice (female, purchased from Experimental Animal Center of Southern Medical University). When the average tumor size reached $\sim 100 \mathrm{~mm}^{3}$, mice with tumor xenografts were randomized into three groups with six mice in each group including: i) vehicle alone (normal saline with $1 \%$ DMSO i.p.), ii) low dose UA (100 mg/kg twice a week i.p.), iii) high dose UA $(200 \mathrm{mg} / \mathrm{kg}$ twice a week i.p.). All of the treatments were administered at a dose of $0.1 \mathrm{ml} / 10 \mathrm{~g}$ bodyweight. The formula, (length $\mathrm{x}$ width $\left.{ }^{2}\right) / 2$, was used to estimate tumor sizes. Both the body weight and tumor sizes were recorded twice a week. At the end of the experiment, the animals were sacrificed and the tumors were dissected and weighed to calculate the inhibitory rate. Animal studies were performed according to the institutional guidelines of Experimental Animal Center in Sun Yat-sen University.

The paraffin tissue sections were obtained from the tumor tissues from the nude mice, IHC were performed as described previously (24). Briefly, the sections were deparaffinizated with xylene and microwaved for $10 \mathrm{~min}$ in the presence of $10 \mathrm{mM}$ citric acid buffer. After quenching the endogenous peroxidases by hydrogen peroxide, the sections were blocked by $5 \%$ BSA/0.3\% Triton X-100 for $20 \mathrm{~min}$. Then the sections were incubated with cleaved caspase-3 (1:200) antibody overnight at $4^{\circ} \mathrm{C}$ and secondary antibody, goat anti-rabbit $\operatorname{IgG}$ (1:50, Proteintech) for $30 \mathrm{~min}$ at $37^{\circ} \mathrm{C}$, and then signal was generated by using the DAB substrate kit (Boster, Wuhan, China) for peroxidase.

Statistical analysis. Data are expressed as mean \pm standard deviation (SD) of three separate experiments and analyzed by SPSS. $\mathrm{P}<0.05$ was considered significant and determined by the two-sample Student's t-test or one-factor ANOVA analysis. 


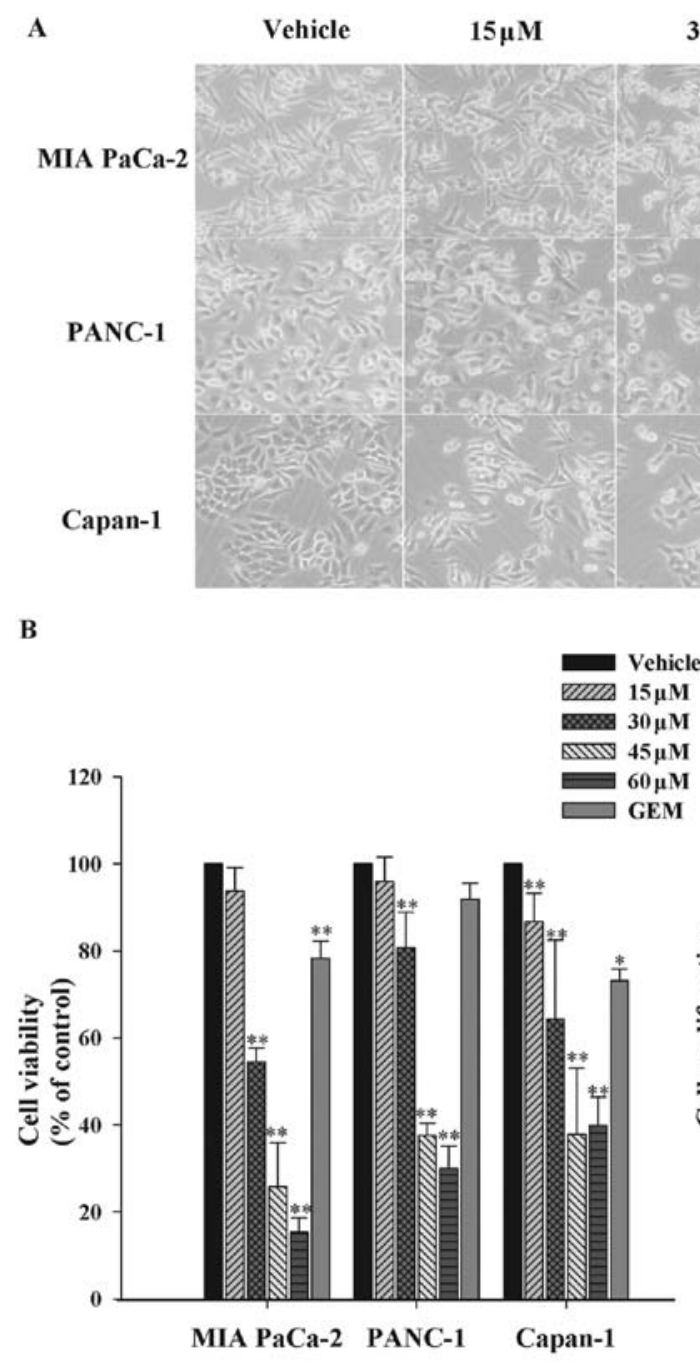

$30 \mu \mathrm{M} \quad 45 \mu \mathrm{M}$

$60 \mu \mathrm{M}$

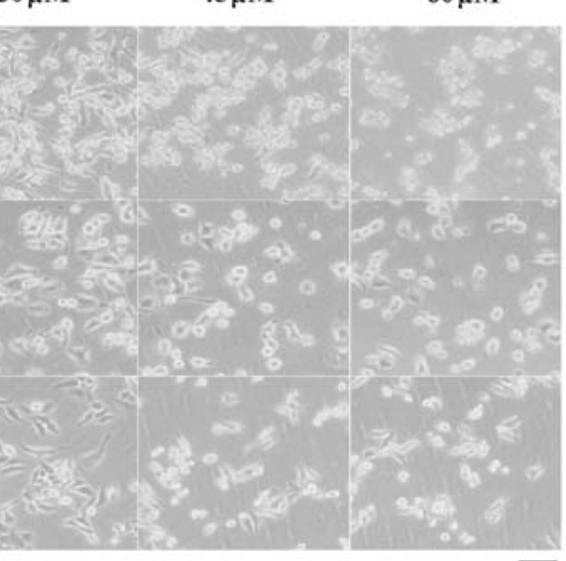

C

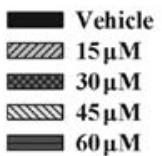

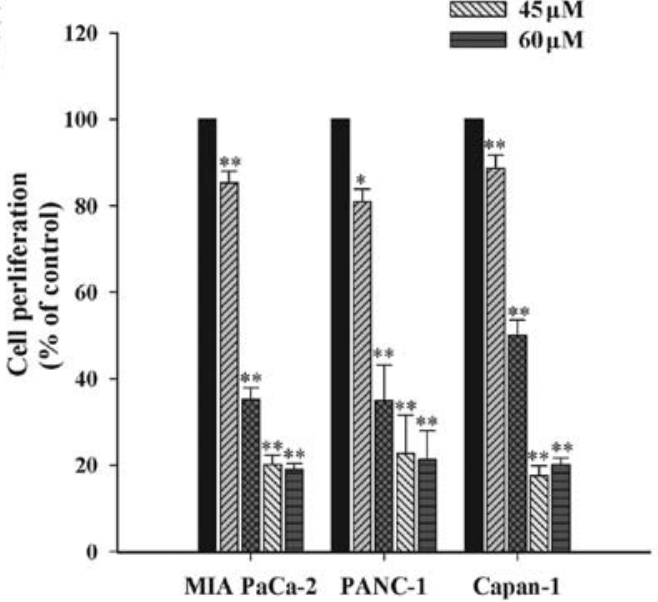

Figure 1. Ursolic acid (UA) dose-dependently decreases the cell viability and proliferation in three different malignancy grades of pancreatic resistant cancer cell lines (MIA PaCa-2, PANC-1 and Capan-1). (A) The effect of UA on cell morphology. The cells were treated with 15, 30,45 and $60 \mu \mathrm{M}$ UA for $24 \mathrm{~h}$. Scale bar, $50 \mu \mathrm{m}$. (B) The effect of UA on cell viability by MTT assay. (C) The effect of UA on cell proliferation by the colorimetric 5'-bromo-2'-deoxy-uridine (BrdU) incorporation assay. Mean $\pm \mathrm{SD}(\mathrm{n}=3) .{ }^{* *} \mathrm{P}<0.05$ vs vehicle; ${ }^{* *} \mathrm{P}<0.01$ vs vehicle.

\section{Results}

Ursolic acid (UA) decreases the viability and proliferation of the human pancreatic resistant cancer cell lines. In order to appraise the cytotoxicity of UA on pancreatic-resistant carcinoma, three different malignancy grades of pancreatic resistant cancer cell lines including MIA PaCa-2 (grade 3), PANC-1 (grade 2) and Capan-1 (grade 1) were exposed to increasing concentrations of $\mathrm{UA}(15,30,45$ and $60 \mu \mathrm{M})$ for $24 \mathrm{~h}(6,25-27)$. Firstly, the morphology of three cell lines under microscopic observation revealed the corresponding reduction of surviving cell numbers and morphological changes after UA treatments (Fig. 1A). In addition, cell viability was analyzed by MTT assay. As shown in Fig. 1B, UA dose-dependently decreased the viability of three cell lines with the half maximal inhibitory concentration $\left(\mathrm{IC}_{50}\right.$ value) at $40.8,45.3$ and $41.3 \mu \mathrm{M}$, respectively. Gemcitabine as the positive control at the concentration of $45 \mu \mathrm{M}$ did not induce significant cytotoxicity to PANC-1 cells comparing with the vehicle for $24 \mathrm{~h}$. However, UA decreased the viability by $62.5 \%$ at the same concentration in the identical cell lines. Similarly,
UA achieved stronger cytotoxic effect than gemcitabine in MIA PaCa-2 and Capan-1 cells. The proliferation inhibition of UA was further investigated by 5'-bromo-2'-deoxy-uridine (BrdU) incorporation assay. As observed in Fig. 1C, growth inhibition effect of UA in MIA PaCa-2 cells started at $15 \mu \mathrm{M}$ and increased up to $60 \mu \mathrm{M}$. The results were in agreement with the outcomes of MTT assay. These data suggested that UA was capable of causing growth inhibition to pancreatic-resistant carcinoma in vitro.

Both intrinsic and extrinsic pathways are involved in UA-triggered apoptosis. UA has been shown to induce apoptosis in multiple cancer cell lines (16-20). To identify whether UA has the same effect in pancreatic-resistant cancer cell lines, the morphologic analysis was performed by Hoechst 33258 staining. MIA PaCa-2, PANC-1 and Capan-1 cells were exposed to various concentrations $(15,30,45$ and $60 \mu \mathrm{M})$ of UA for $24 \mathrm{~h}$, the morphological characteristics of apoptotic cells such as condensed nuclei and cell shrinkage were discovered and apoptotic proportion grew with upward dose (Fig. 2A). To confirm the UA-triggered apoptosis in three cell lines, another 


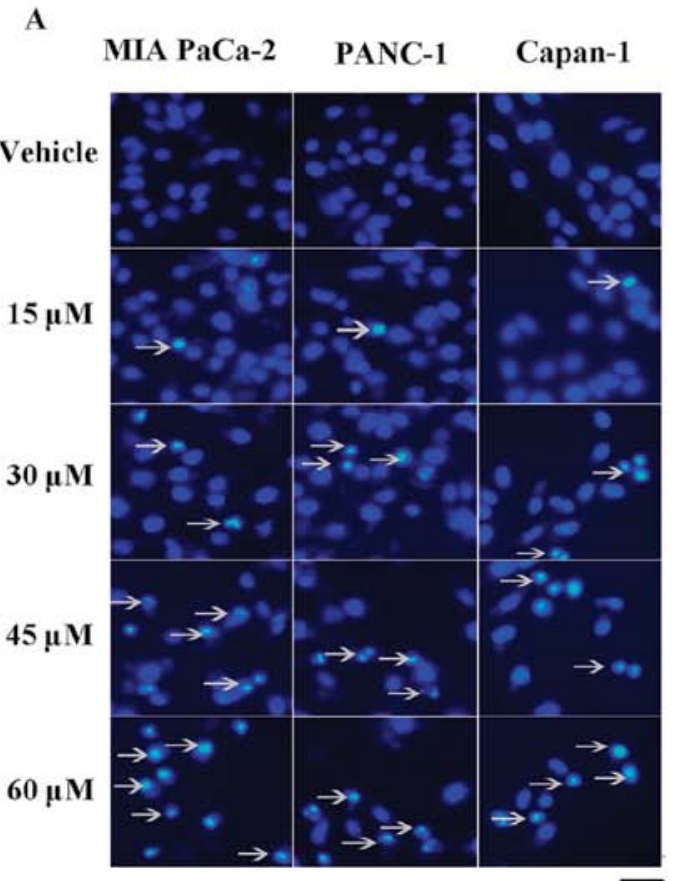

c

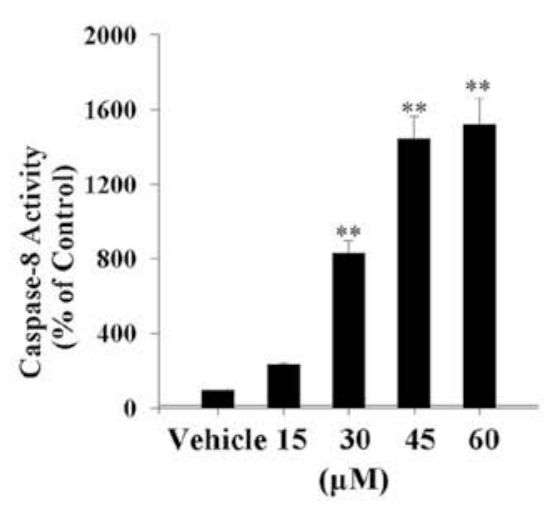

D

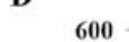

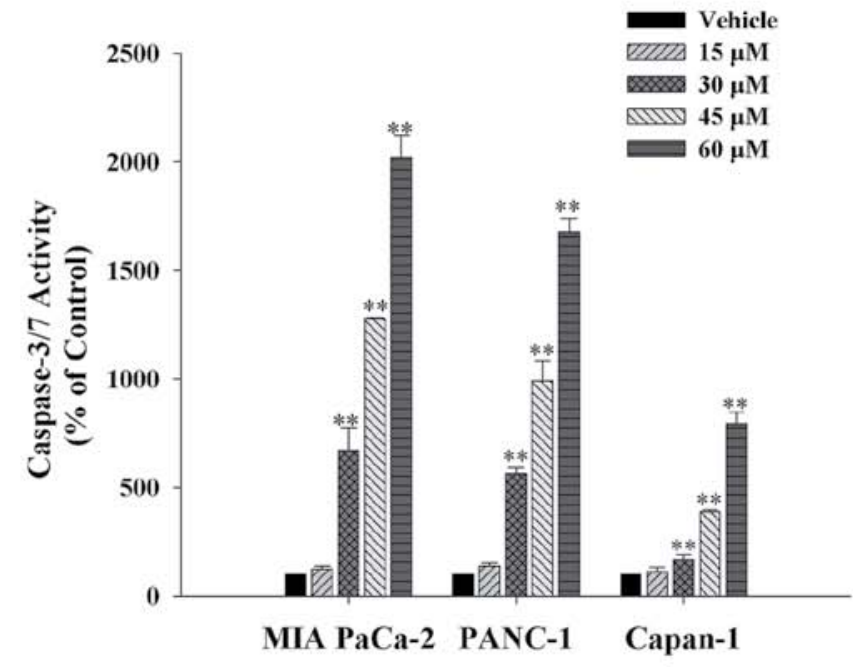

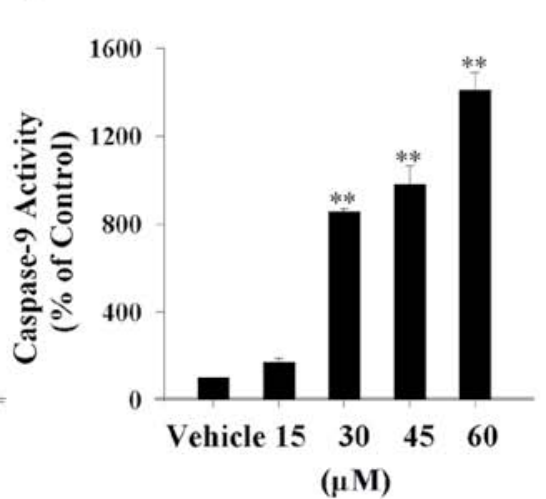

Figure 2. Ursolic acid (UA)-triggered apoptosis is mediated via the intrinsic and extrinsic pathways in pancreatic resistant cancer cell lines. (A) Hoechst 33258 staining. MIA PaCa-2, PANC-1 and Capan-1 cells were incubated with 15, 30, 45 and $60 \mu \mathrm{M}$ UA for $24 \mathrm{~h}$. Scale bar, $50 \mu \mathrm{m}$. Arrows indicate the nuclei of apoptotic cells. (B-E) The effect of UA on (B) the caspase-3/7, (C) the caspase- 8 activity, (D) the cytochrome $\mathrm{C}$ release and (E) the caspase- 9 activity in MIA PaCa-2 cells. Mean $\pm \operatorname{SD}(\mathrm{n}=3){ }^{* * *} \mathrm{P}<0.01$ vs vehicle.

apoptotic marker, caspase-3/7 activity, was examined. Clearly, the caspase- $3 / 7$ activity was promoted gradually along with the rising concentrations of UA. Fig. 2B shows that the caspase-3/7 activity was enhanced 20-, 16- and 8-fold, respectively, in MIA PaCa-2, PANC-1 and Capan-1 cells at $60 \mu \mathrm{M}$ UA.

The caspase-dependent cascade is mediated by endogenous and exogenous signal transductions (22). To study by which UA induces caspase- $3 / 7$ activation, several markers belonging to the two pathways were inspected. Caspase- 8 is a downstream apoptotic molecule of the death receptor, which mediates the death receptor signaling pathway (extrinsic pathway). As shown in Fig. 2C, caspase-8 activity increased by $\sim 15$-fold with $60 \mu \mathrm{M}$ UA treatment. Cytochrome $\mathrm{C}$ released from mitochondria into cytoplasm was the initial step in the intrinsic apoptotic pathway. Fig. 2D exhibits the cytochrome $\mathrm{C}$ relative release rate increase by $\sim 4.5$-fold at $60 \mu \mathrm{M}$ UA. Besides, caspase- 9 activity, another marker of the intrinsic apoptotic pathway, was also increased by $\sim 14$-fold after $60 \mu \mathrm{M}$ UA intervention (Fig. 2E).
c-Jun-terminal kinase (JNK) pathway is potentially involved in UA-induced endogenous apoptosis. The mitogen-activated protein kinase (MAPK) family is closely related to cell apoptosis (28). There are some reports revealing that UA is able to activate JNK, which is one of crucial components in MAPK signaling pathway $(16,29)$. Here, we detected JNK and P38 expression levels, both of which were reported as vital molecules of MAPK family in the programmed cell death, to explore more potential signal channels (30). We found that JNK activity, measured by phosphorylated-JNK level, was significantly up-regulated at the presence of 30 and $45 \mu \mathrm{M}$ UA. But, UA had no obvious effect on JNK, P38 and phosphorylated-P38 protein levels (Fig. 3A). This result indicated that JNK may contribute to UA-induced apoptosis. To further study the apoptotic pathway in which JNK was involved, we used the JNK inhibitor SP600125 to block the activity of JNK pathway. MIA PaCa-2 cells were pretreated with $10 \mu \mathrm{M}$ SP600125 for $1 \mathrm{~h}$, and then treated with $45 \mu \mathrm{M}$ UA for $12 \mathrm{~h}$. As shown in Fig. 3B and C, pretreatment 
A

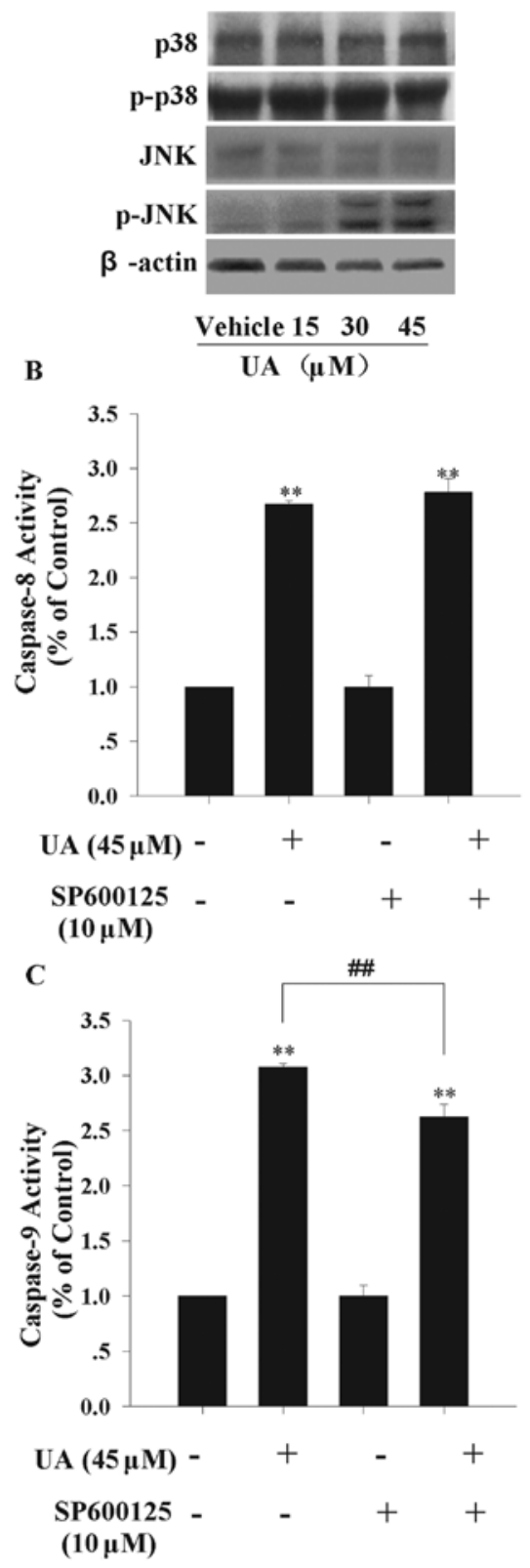

Figure 3. c-Jun-terminal kinase (JNK) pathway is potentially involved in ursolic acid (UA)-induced endogenous apoptosis in MIA PaCa-2 cells. (A) The effect of UA on the protein levels of JNK, phosphorylated-JNK, P38 and phosphorylated-P38 by western blotting. MIA PaCa-2 cells were incubated with $15,30,45 \mu \mathrm{M}$ UA for $12 \mathrm{~h}$. (B) The effect of $45 \mu \mathrm{M}$ UA on the caspase- 8 and (C) the caspase- 9 activity at $12 \mathrm{~h}$ after the pretreatment of the JNK inhibitor SP600125 $(10 \mu \mathrm{M})$ for $1 \mathrm{~h}$. Mean $\pm \mathrm{SD}(\mathrm{n}=3) .{ }^{* * *} \mathrm{P}<0.01$ vs vehicle; ${ }^{\# \#} \mathrm{P}<0.01$ vs $\mathrm{UA} 45 \mu \mathrm{M}$

by SP600125 partially inhibited the caspase- 9 activity by $16 \%$, while caspase- 8 activity was not altered significantly. These data indicated that JNK is at least partly involved in UA-induced endogenous apoptotic signaling transduction.

UA inhibits the PI3K/Akt pathway and down-regulates the expression of downstream proteins. To ascertain the potential mechanism by which UA caused growth suppression and apoptosis to pancreatic cancer, we also examined the activity of PI3K/Akt pathway in MIA PaCa-2 cell line. When cells were in the presence of increasing concentrations of UA (15,
30 and $45 \mu \mathrm{M}$ ) for $12 \mathrm{~h}$, the results by western blotting demonstrated the total PI3K and the phosphorylated Akt levels were attenuated gradually in a dose-dependent manner, while the total Akt protein expressions did not alter (Fig. 4A). PI3K/Akt pathway is constitutively activated in most tumor cell lines and regulates a series of biological characteristics of tumors such as growth and apoptosis (31). Therefore, we checked the possible Akt targeting effector molecules downstream. Similar changes to Akt, were recorded for the phosphorylation level of GSK-3 $\beta$, MDM2, P65, IкB that also decreased gradually, whereas the total proteins of GSK-3 $\beta$, P65, I $\kappa$ B did not show any corresponding alteration (Fig. 4B).

PI3K/Akt/NF- $\kappa B$ pathway is involved in UA-induced cell viability decrease, but not intrinsic and extrinsic apoptosis. Aberrant activation of NF- $\kappa \mathrm{B}$ greatly contributes to chemoresistance in pancreatic cancer, and suppression of $\mathrm{NF}-\kappa \mathrm{B}$ sensitizes the treatment outcome of gemcitabine (32). To assess whether the UA-induced NF- $\kappa \mathrm{B}$ activation is a downstream event of $\mathrm{PI} 3 \mathrm{~K} / \mathrm{Akt}$ signaling pathway, I $\mathrm{B}$ and its phosphorylated form were further investigated. We inhibited the phosphorylation of Akt by 10, 20, $40 \mu \mathrm{M}$ LY294002 for $12 \mathrm{~h}$. As seen in Fig. 4C, $20 \mu \mathrm{M}$ LY294002 and above abolished more than half of the phosphorylated level of Akt protein. Phosphorylated $\mathrm{I} \kappa \mathrm{B}$ was evidently modulated downward in a dose-dependent pattern. However, there was no alteration of total $\mathrm{I} \kappa \mathrm{B}$ within $12 \mathrm{~h}$. In addition, the nuclear translocation of the cytoplasmic NF- $\kappa \mathrm{B}$ p65 subunit was also checked by immunofluorescence. UA $(45 \mu \mathrm{M})$ and/or $40 \mu \mathrm{M} \mathrm{LY} 294002$ for $12 \mathrm{~h}$ repressed the NF- $\kappa \mathrm{B}$ p65 nuclear translocation compared with vehicle (Fig. 4D). These results suggested there was crosstalk between PI3K/Akt and NF- $\kappa \mathrm{B}$ signaling pathways in MIA PaCa- 2 cells.

We next examined the involvement of PI3K/Akt/NF- $\mathrm{BB}$ pathway in the apoptotic cascade. The MIA PaCa-2 cells were incubated with $40 \mu \mathrm{M} \mathrm{LY} 294002$ for $12 \mathrm{~h}$. As seen in Fig. 4E and F, inhibiting the activation of Akt can not activate caspase- 8 and -9 . Hence, PI3K/Akt/NF- $\kappa$ B pathway is not the main participant in UA-induced intrinsic and extrinsic apoptotic signaling transductions. However, the cell viability was decreased by $\sim 25 \%$ after exposure to $40 \mu \mathrm{M} \mathrm{LY} 294002$ for $24 \mathrm{~h}$. These findings indicated that PI3K/Akt/NF- $\mathrm{B}$ pathway was involved in UA-induced cytotoxicity to MIA PaCa-2 cells, but the mechanism was not caspase-8- and -9-dependent.

UA overcomes chemoresistance in other resistant cancer cell lines. We also tested whether UA possessed the potential to induce other resistant cancer cells death in the similar fashion. A paclitaxel-resistant endometrial cell line (HEC-1A) and a platinum-resistant ovarian cancer cell line (OVCAR-3) were used to examine the cell viability after UA exposure $(33,34)$. As seen in Fig. 5A and B, UA inhibited the cell viability in a time and dose-dependent manner. Moreover, UA also increased the phosphorylated JNK, and decreased the expression of phosphorylated Akt (Fig. 5C and D). These results further confirmed that the mechanism of action of UA was associated with JNK and Akt pathways.

UA causes tumor regression in MIA PaCa-2 xenograft model. The data described above clearly showed that UA was a potent compound to inhibit proliferation and caused deaths of various 


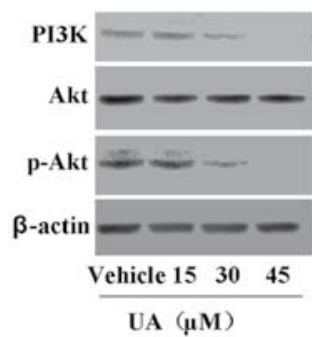

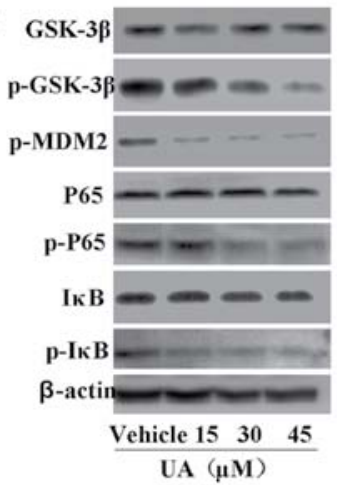

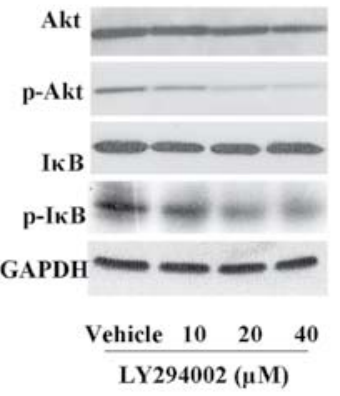

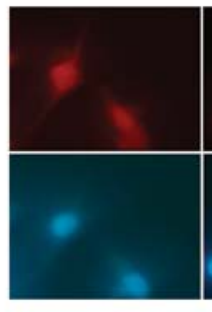
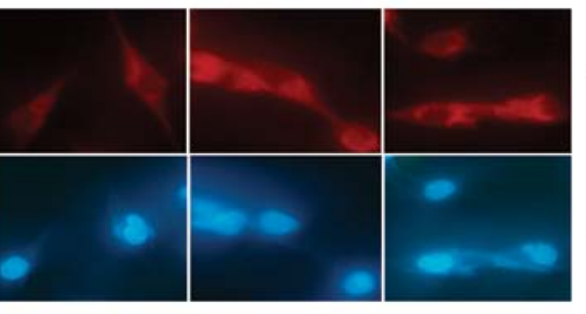

NF-k B P65

Vehicle

$\mathrm{UA} 45 \mu \mathrm{M}$

LY294002 $40 \mu \mathrm{M}$ Combination

DAPI

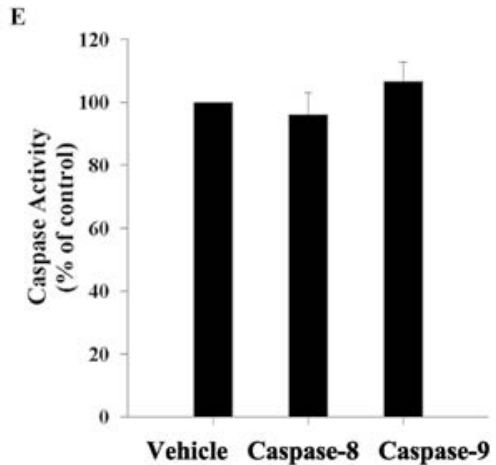

$\mathbf{F}$

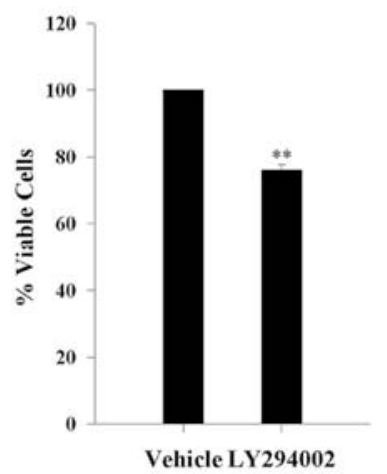

Figure 4. PI3K/Akt/NF-kB pathway is involved in ursolic acid (UA)-induced cell viability decreases, but not intrinsic and extrinsic apoptosis in MIA PaCa-2 cells (A) The effect of UA on the protein levels of PI3K, Akt and phosphorylated-Akt by western blotting. MIA PaCa-2 cells were incubated with $15,30,45 \mu \mathrm{M}$ UA for $12 \mathrm{~h}$. (B) The effect of UA on the downstream protein levels of PI3K/Akt pathway at $12 \mathrm{~h}$. (C) The effect of Akt inhibitor LY294002 on IкB and phosphorylated-IKB expressions by western blotting. MIA PaCa-2 cells were treated with 10, 20,40 $\mu \mathrm{M} \mathrm{LY} 294002$ for $12 \mathrm{~h}$. (D) The effect of Akt inhibitor $40 \mu \mathrm{M} \mathrm{LY} 294002$ on NF- $\mathrm{kB}$ p65 nuclear translocation at $12 \mathrm{~h}$. (E) The effect of $40 \mu \mathrm{M} \mathrm{LY} 294002$ on the caspase-8 and -9 activity at $12 \mathrm{~h}$. Scale bar, $10 \mu \mathrm{m}$. (F) The effect of $40 \mu \mathrm{M} \mathrm{LY} 294002$ on the cell viability at $24 \mathrm{~h}$. Mean $\pm \mathrm{SD}(\mathrm{n}=3) .{ }^{* *} \mathrm{P}<0.01$ vs vehicle.
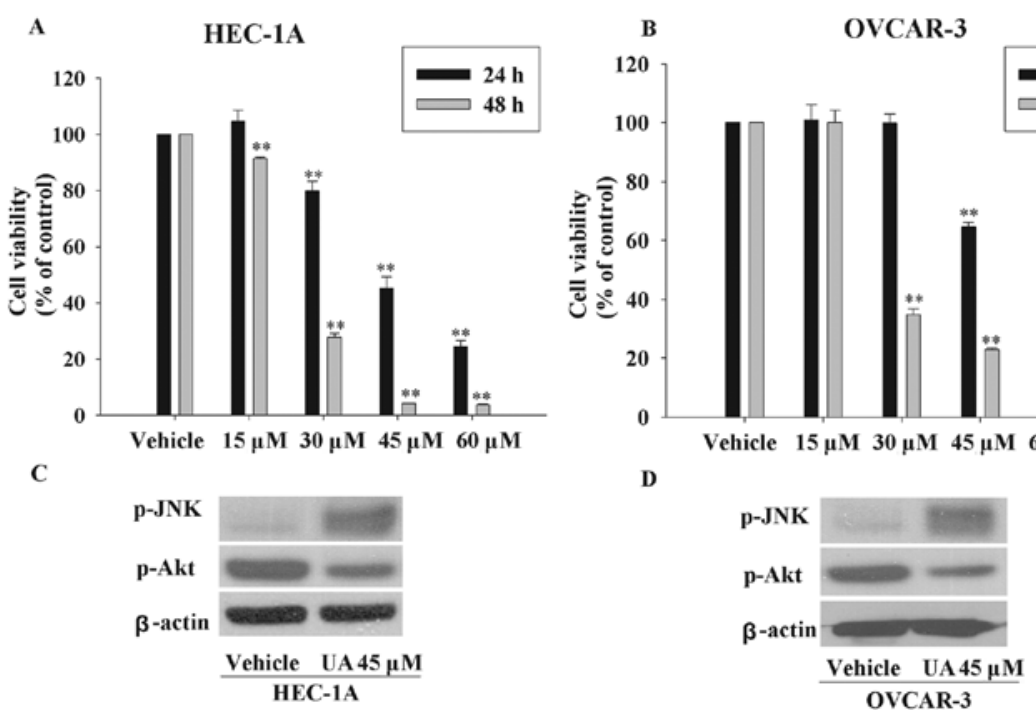

Figure 5. Ursolic acid (UA) could overcome chemoresistance in other resistant cancer cell lines. (A and B) The effect of UA on cell viability in HEC-1A and OVCAR-3 cell lines. The two cell types were treated with 15, 30, 45, $60 \mu \mathrm{M}$ UA for $24 \mathrm{~h}$ and the viability was examined by MTT assay. (C and D) The effect of $\mathrm{UA}$ on the protein levels of phosphorylated-JNK, and phosphorylated-Akt by western blotting. Mean $\pm \mathrm{SD}(\mathrm{n}=3) .{ }^{* *} \mathrm{P}<0.01$ vs vehicle. 
A

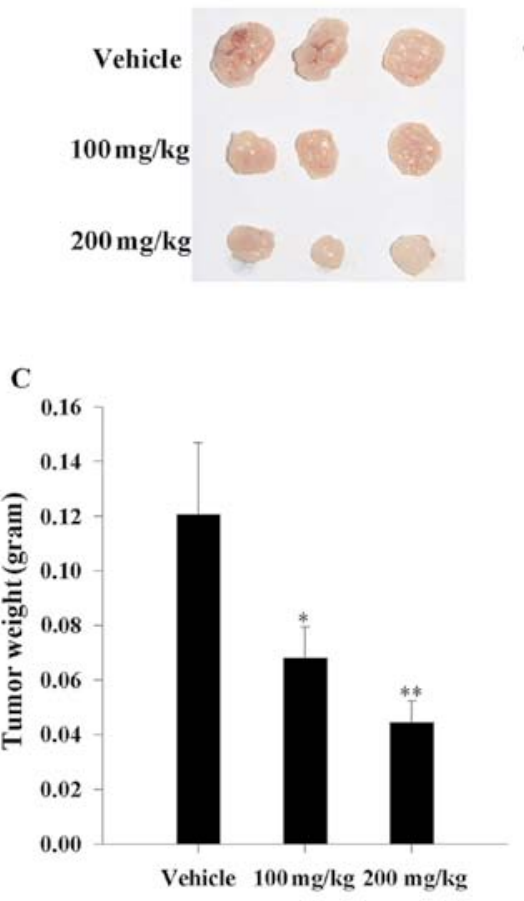

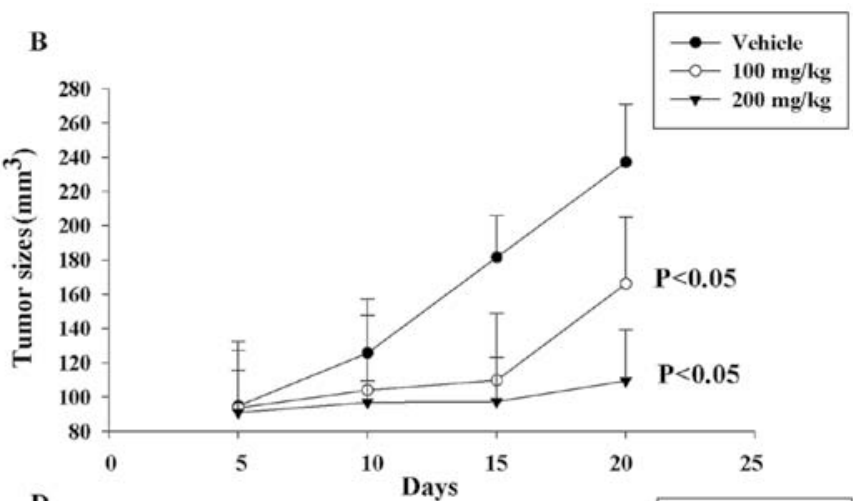

D

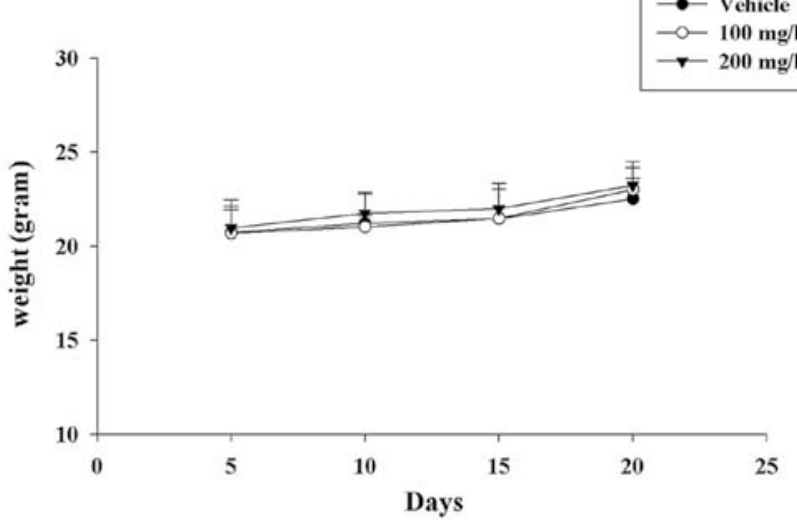

E

Cleavage caspase-3

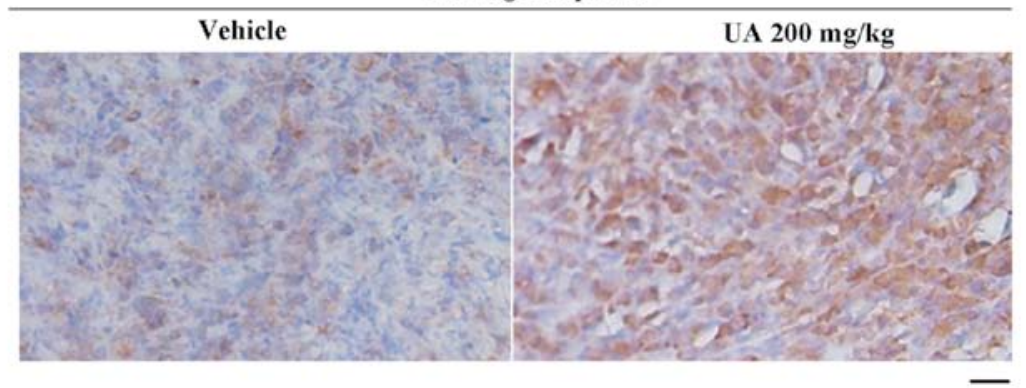

Figure 6. Ursolic acid (UA) suppresses the gemcitabine-resistant pancreatic cancer in vivo. (A) UA caused pancreatic tumor regression in vivo. MIA PaCa-2 cells $\left(5 \times 10^{6}\right)$ were injected into the flanks of 6-week-old nude mice. When the average tumor size reached $\sim 100 \mathrm{~mm}^{3}$, mice were randomly divided into three groups with six mice in each group and treated with saline with $1 \%$ DMSO, UA at 100 and $200 \mathrm{mg} / \mathrm{kg}$ twice a week, respectively. (B) The growth curve of xenografts in mice. (C) The tumor weight of xenografts in mice after the end of UA treatment. (D) The body weight curve of mice to evaluate the toxicity of UA. (E) The effect of UA on activity of the cleavage caspase- 3 in vivo by immunohistochemistry. Scale bar, $50 \mu \mathrm{m}$. Mean $\pm \mathrm{SD}$. " $\mathrm{P}<0.05$ vs vehicle; ${ }^{* *} \mathrm{P}<0.01$ vs vehicle.

chemotherapy-resistant cancer cell lines. Thus we tried to apply UA in a nude mouse xenograft model to provide evidence for clinical application. Until the tumor volume reached $\sim 100 \mathrm{~mm}^{3}$, the mice were treated i.p. with either vehicle or UA at $100 / 200 \mathrm{mg} / \mathrm{kg}$ twice a week. Significant inhibition of tumor growth by UA was observed after UA treatment for 20 days (Fig. 6A and B). Tumor weights in UA-treated groups were also lower than the vehicle group (30\% less in $100 \mathrm{mg} / \mathrm{kg}$ group and $50 \%$ less in $200 \mathrm{mg} / \mathrm{kg}$ group) (Fig. 6C). In order to measure the safety of UA, body weights of mice were regularly detected. As seen in Fig. 6D, there were no significant differences compared with control group after the UA treatment. Furthermore, we checked the cleavage caspase- 3 expression by immunohistochemistry to reveal that the tumor in UA-exposed group had more visible apoptosis. These data elucidated that UA caused resistant pancreatic cancer death also in vivo.

\section{Discussion}

Due to the poor early diagnosis and serious chemotherapy resistance, pancreatic carcinoma is highly fatal. Although gemcitabine is known as the most active chemotherapy agent for this tumor, it has only a slight survival benefit and shows an objective tumor response rate of $<10 \%$ owing to pre-existing or acquired chemoresistance $(35,36)$, thus demanding urgent development of effective therapeutic options. Here we demonstrate that a small molecule ursolic acid (UA), a well known anti-oxidative and anti-inflammatory agent, significantly suppresses the growth and induces apoptosis of resistant pancreatic cancer through activation of c-Jun-terminal kinase (JNK) pathway and inhibition of $\mathrm{PI} 3 \mathrm{~K} / \mathrm{Akt} / \mathrm{NF}-\kappa \mathrm{B}$ pathway.

UA, belonging to pentacyclic triterpenoid, is a soluble, natural, and small molecule compound, which has displayed 
its proliferation-inhibiting and apoptosis-inducing effects in some chemotherapy-resistant tumors $(16,17,37)$. Previous study showed that UA only decreased the cell viability by $\sim 40 \%$ in a hormone refractory prostate cancer cell line at $72 \mathrm{~h}$ (16). In contrast to the prostate cancer cells, UA exhibits much more potent cytotoxicity to pancreatic tumors. The half maximal inhibitory concentration ( $\mathrm{IC}_{50}$ value) of UA was $40.8 \mu \mathrm{M}$ in highly malignant pancreatic cancer cells (MIA PaCa-2 cells) within $24 \mathrm{~h}$ in vitro. In our data, both modulation of proliferation and induction of apoptosis by UA were observed within the time course of $24 \mathrm{~h}$. However, UA had suppressed the proliferation significantly at $15 \mu \mathrm{M}$ rather than any type of caspase activity or cytochrome $\mathrm{C}$ relative release rate. The alterations of proliferation inhibiting rate and caspase-3/7 activity were detected at 30-45 $\mu \mathrm{M}$ UA. Furthermore, UA at the higher concentration $(60 \mu \mathrm{M})$ was only perceived to be effective in apoptosis induction. Hence, the results seem to indicate that decreased proliferation and increased apoptosis by UA together impact cell viability of pancreatic cancer cells.

The JNK pathway reported as important apoptosis inducing factor exerts antagonistic effects on apoptosis (30). JNK, a mitogen-activated protein kinase (MAPK), can be activated primarily by exposure to environmental stress (38). Although the extracellular signal-regulated kinase (ERK) pathway is considered as a great contributor to oncogenesis, previous study has demonstrated that it plays a lesser role in mitogen-induced survival of pancreatic cancer (39). However, many studies have reported that JNK pathway plays a crucial role in UA-induced apoptosis in many cancers $(16,29,40)$. In our study, the activation of $\mathrm{JNK}$, but not P38, was induced by UA in a dosedependent manner in MIA PaCa-2 cells. Two major apoptotic signaling transductions, namely the receptor-mediated/exogenous and mitochondrial/endogenous pathways, are activated in UA-induced apoptosis in MIA PaCa- 2 cells. The trigger of endogenous pathway depends on the release of cytochrome $\mathrm{C}$ from mitochondria to cytosol and activation of caspase-9 (41). In the extrinsic pathway, the binding of death ligands to their homologous receptors drives activity of caspase-8 (42). Here, we showed that SP600125, a specific JNK inhibitor, could partly protect UA-induced caspase-9 activation rather than caspase-8. Our data are consistent with a previous report (16). Teraishi et al (43) have proved that JNK activation is required for gemcitabine to achieve its cytotoxicity and the weaker activity of JNK may result in gemcitabine resistance. Our data displayed that JNK pathway was activated excessively in the gemcitabine resistant cell line (MIA $\mathrm{PaCa}-2$ ). We proved similarly that JNK pathway participated in UA-induced mitochondrial apoptotic signaling transduction in MIA $\mathrm{PaCa}-2$ cells. Thus we speculate that UA-induced JNK activation may contribute to overcome gemcitabine resistance and induces programmed cell death in gemcitabine resistant pancreatic cancer.

The current consensus to PI3K/Akt signaling transduction is that this cascade contributes greatly to tumor progression and chemotherapy resistance (44). PI3K is a broadly expressed lipid kinase, the main action of which is to execute the catalyzed reaction to synthesize the second messenger-PIP $\mathrm{P}_{3}$ from $\mathrm{PIP}_{2}$ $(44,45)$. Akt, acting downstream of PI3K, is usually activated by directly binding to PIP3 through its pleckstrin-homology (PH) domain and phosphorylation (44). Akt activation is closely relevant to anti-apoptosis and proliferative signal transduction. Primarily, Akt stimulates the degradation of $\mathrm{I} \kappa \mathrm{B}$ which results in the nuclear translocation of P65 and activation of target genes (46). Akt also catalyzes phosphorylation of MDM to improve its function to bind to P53 and enhance p53 degradation $(47,48)$. In the aspect of cell proliferative promotion, Akt inhibits the kinase activity of GSK3 $\beta$ by maintaining the phosphorylated level to protect cyclin D from degradation (49). Our results showed that UA strongly suppressed the activation of Akt and blocked the activity of its downstream molecules mentioned above.

We proved that suppressing the Akt activity by LY294002, a selective Akt inhibitor, down-regulated the NF- $\kappa \mathrm{B}$ activation by reducing the p65 nuclear translocation and phosphorylating I $\mathrm{B}$ in MIA PaCa- 2 cells. We propose that PI3K/Akt pathway seems crosstalk with NF- $\mathrm{B}$ pathway in pancreatic cancer cells. Arlt $e t$ al (32) has reported previously the evident correlation between basal NF- $\kappa \mathrm{B}$ activity and gemcitabine resistance in pancreatic cancer cells. There is some evidence showing that PI3K/Akt inhibition promoted anti-tumor effectiveness of gemcitabine via inhibiting PI3K/Akt phosphorylation in vitro and in vivo $(50,51)$. UA may demonstrate its potent anti-cancer effects via PI3K/ Akt/NF- $\kappa \mathrm{B}$ pathway in MIA PaCa-2 cells. We observed that LY294002 did not protect MIA PaCa-2 cells from caspase-8/9 activations. Therefore, exogenous and endogenous apoptosis are not affected equally by UA-triggered Akt activation. However, LY294002 decreased the viability of MIA PaCa-2 cells to some extent, and it is well-known that cell viability is a combination of cell proliferation and death. We argue that the UA-induced $\mathrm{PI} 3 \mathrm{~K} / \mathrm{Akt} / \mathrm{NF}-\kappa \mathrm{B}$ pathway suppression may only affect the pancreatic cancer cell proliferative rate. Besides, recent study also demonstrated that UA was able to induce tumor cells apoptosis through a caspase-independent pathway (17). That phenomenon means UA has the chance to apply for combined treatment to overcome chemotherapy resistance. In our study, we can not exclude that the PI3K/Akt/NF- $\kappa \mathrm{B}$ pathway also triggers caspase-independent apoptosis. But further studies are needed to prove the hypothesis.

To examine whether the effect of UA on JNK and Akt pathway is confined to resistant pancreatic cancer cells solely or is a general phenomenon which is applicable to other resistant cancer cells, we examined the effect of UA on an endometrial cell line (HEC-1A) and an ovarian cancer cell line (OVCAR-3) because both of them are highly resistant to chemotherapy. According to our data, the JNK activity was inhibited, but Akt was excessively activated in these cells. UA significantly decreased the cell viability of HEC-1A cells and OVCAR-3 cells, which was associated with an increase of JNK activation and a decrease of Akt activation. For future clinical application, the safety and efficacy of UA were further detected in vivo. We presented evidence that UA suppressed tumor growth without any obvious side effects. As a natural compound extracted from edible foods and herbs, UA is relatively low-toxic with $\mathrm{LD}_{50}$ value $>637 \mathrm{mg} / \mathrm{kg}$ intraperitoneally in mice. Our studies provide further evidence that UA is a potent compound to apply to overcome chemoresistance in oncological therapy.

In conclusion, UA is a small, safe, potent and multifuntional molecule which is easily produced and relatively safe. UA has been applied in clinical trial in some areas to treat cancer (52). Consequently, UA is comparable or even superior to conventional anti-tumor drugs, encouraging further the use of this small 
molecular compound in gemcitabine resistant pancreatic cancer and other chemo-insensitive cancers.

\section{Acknowledgements}

This study was supported by National Natural Science Foundation of China (no. 30973202), Doctoral Fund of Ministry of Education of China (no. 20090171110059), Natural Science Foundation of Guangdong Province (no. S2011010004621), and Guangdong Provincial Fund of Industry, Education and Academy (no. 2008B090500194).

\section{References}

1. Stathis A and Moore MJ: Advanced pancreatic carcinoma: current treatment and future challenges. Nat Rev Clin Oncol 7: 163-172, 2010.

2. Campbell PJ, Yachida S and Mudie LJ, et al: The patterns and dynamics of genomic instability in metastatic pancreatic cancer. Nature 467: 1109-1113, 2010.

3. Herrmann R and Jelic S: Pancreatic cancer: ESMO clinical recommendations for diagnosis, treatment and follow-up. Ann Oncol 19 (Suppl 2): I25-I26, 2008.

4. Rosewicz S and Wiedenmann B: Pancreatic carcinoma. Lancet 349: 485-489, 1997.

5. Burris HR, Moore MJ, Andersen J, et al: Improvements in survival and clinical benefit with gemcitabine as first-line therapy for patients with advanced pancreas cancer: a randomized trial. J Clin Oncol 15: 2403-2413, 1997.

6. Ou YQ, Zhu W, Li Y, et al: Aspirin inhibits proliferation of gemcitabine-resistant human pancreatic cancer cells and augments gemcitabine-induced cytotoxicity. Acta Pharmacol Sin 31: 73-80, 2010.

7. Ina S, Hirono S, Noda $\mathrm{T}$ and Yamaue $\mathrm{H}$ : Identifying molecular markers for chemosensitivity to gemcitabine in pancreatic cancer: increased expression of interferon-stimulated gene $15 \mathrm{kDa}$ is associated with intrinsic chemoresistance. Pancreas 39: 473-485, 2010.

8. Jemal A, Siegel R, Ward E, Murray T, Xu J and Thun MJ: Cancer statistics, 2007. CA Cancer J Clin 57: 43-66, 2007.

9. Liu J: Oleanolic acid and ursolic acid: research perspectives. J Ethnopharmacol 100: 92-94, 2005.

10. Liu J: Pharmacology of oleanolic acid and ursolic acid. J Ethnopharmacol 49: 57-68, 1995.

11. Shih YH, Chein YC, Wang JY and Fu YS: Ursolic acid protects hippocampal neurons against kainate-induced excitotoxicity in rats. Neurosci Lett 362: 136-140, 2004.

12. Chung YK, Heo HJ, Kim EK, et al: Inhibitory effect of ursolic acid purified from Origanum majorana $\mathrm{L}$ on the acetylcholinesterase. Mol Cells 11: 137-143, 2001.

13. Tsai SJ and Yin MC: Antioxidative and anti-inflammatory protection of oleanolic acid and ursolic acid in PC12 cells. J Food Sci 73 H174-H178, 2008.

14. Somova LO,Nadar A, Rammanan Pand Shode FO: Cardiovascular, antihyperlipidemic and antioxidant effects of oleanolic and ursolic acids in experimental hypertension. Phytomedicine 10: 115-121, 2003.

15. Cha HJ, Bae SK, Lee HY, et al: Anti-invasive activity of ursolic acid correlates with the reduced expression of matrix metalloproteinase-9 (MMP-9) in HT1080 human fibrosarcoma cells. Cancer Res 56: 2281-2284, 1996.

16. Zhang Y, Kong C, Zeng Y, et al: Ursolic acid induces PC-3 cell apoptosis via activation of JNK and inhibition of Akt pathways in vitro. Mol Carcinog 49: 374-385, 2010.

17. Yang L, Liu X, Lu Z, et al: Ursolic acid induces doxorubicinresistant HepG2 cell death via the release of apoptosis-inducing factor. Cancer Lett 298: 128-138, 2010.

18. Xavier CP, Lima CF, Preto A, Seruca R, Fernandes-Ferreira M and Pereira-Wilson C: Luteolin, quercetin and ursolic acid are potent inhibitors of proliferation and inducers of apoptosis in both KRAS and BRAF mutated human colorectal cancer cells. Cancer Lett 281: 162-170, 2009.

19. Manu KA and Kuttan G: Ursolic acid induces apoptosis by activating p53 and caspase-3 gene expressions and suppressing NF-kappaB mediated activation of bcl-2 in B16F-10 melanoma cells. Int Immunopharmacol 8: 974-981, 2008.
20. Wang JS, Ren TN and Xi T: Ursolic acid induces apoptosis by suppressing the expression of FoxM1 in MCF-7 human breast cancer cells. Med Oncol 29: 10-15, 2012.

21. Zhu W, Ou Y, Li Y, et al: A small-molecule triptolide suppresses angiogenesis and invasion of human anaplastic thyroid carcinoma cells via down-regulation of the nuclear factor-kappa B pathway. Mol Pharmacol 75: 812-819, 2009.

22. Li J, Zhu W, Leng T, et al: Triptolide-induced cell cycle arrest and apoptosis in human renal cell carcinoma cells. Oncol Rep 25: 979-987, 2011.

23. He D, Xu Q, Yan M, et al: The NF-kappaB inhibitor, celastrol, could enhance the anti-cancer effect of gambogic acid on oral squamous cell carcinoma. BMC Cancer 9: 343, 2009.

24. Stairs DB, Nakagawa H, Klein-Szanto A, et al: Cdx1 and c-Myc foster the initiation of transdifferentiation of the normal esophageal squamous epithelium toward Barrett's esophagus. PLoS One 3: e3534, 2008

25. Xia Y, Liu Y, Wan J, et al: Novel triazole ribonucleoside downregulates heat shock protein 27 and induces potent anticancer activity on drug-resistant pancreatic cancer. J Med Chem 52: 6083-6096, 2009.

26. Jonckheere N, Fauquette V, Stechly L, et al: Tumour growth and resistance to gemcitabine of pancreatic cancer cells are decreased by AP-2alpha overexpression. Br J Cancer 101: 637-644, 2009.

27. Miyamoto H, Murakami T, Tsuchida K, Sugino H, Miyake H and Tashiro S: Tumor-stroma interaction of human pancreatic cancer: acquired resistance to anticancer drugs and proliferation regulation is dependent on extracellular matrix proteins. Pancreas 28 : $38-44,2004$

28. Chang L and Karin M: Mammalian MAP kinase signalling cascades. Nature 410: 37-40, 2001

29. Zhang YX, Kong CZ, Wang LH, et al: Ursolic acid overcomes Bcl-2-mediated resistance to apoptosis in prostate cancer cells involving activation of JNK-induced $\mathrm{Bcl}-2$ phosphorylation and degradation. J Cell Biochem 109: 764-773, 2010.

30. Wagner EF and Nebreda AR: Signal integration by JNK and p38 MAPK pathways in cancer development. Nat Rev Cancer 9: 537-549, 2009.

31. Falasca M: PI3K/Akt signalling pathway specific inhibitors: a novel strategy to sensitize cancer cells to anti-cancer drugs. Curr Pharm Des 16: 1410-1416, 2010.

32. Arlt A, Gehrz A, Muerkoster S, et al: Role of NF-kappaB and Akt/ $\mathrm{PI} 3 \mathrm{~K}$ in the resistance of pancreatic carcinoma cell lines against gemcitabine-induced cell death. Oncogene 22: 3243-3251, 2003.

33. Wang X, Yang Y, Xu C, et al: CHFR suppression by hypermethylation sensitizes endometrial cancer cells to paclitaxel. Int J Gynecol Cancer 21: 996-1003, 2011.

34. Lange TS, Kim KK, Singh RK, Strongin RM, McCourt CK and Brard L: Iron(III)-salophene: an organometallic compound with selective cytotoxic and anti-proliferative properties in platinumresistant ovarian cancer cells. PLoS One 3: e2303, 2008.

35. Welch SA and Moore MJ: Combination chemotherapy in advanced pancreatic cancer: time to raise the white flag? J Clin Oncol 25: 2159-2161, 2007.

36. Mamaghani S,Patel S and Hedley DW: Glycogen synthase kinase-3 inhibition disrupts nuclear factor-kappaB activity in pancreatic cancer, but fails to sensitize to gemcitabine chemotherapy. BMC Cancer 9: 132, 2009.

37. Shan JZ, Xuan YY, Ruan SQ and Sun M: Proliferation-inhibiting and apoptosis-inducing effects of ursolic acid and oleanolic acid on multi-drug resistance cancer cells in vitro. Chin J Integr Med 17: 607-611, 2011.

38. Weston CR and Davis RJ: The JNK signal transduction pathway. Curr Opin Cell Biol 19: 142-149, 2007.

39. Perugini RA, McDade TP, Vittimberga FJ and Callery MP: Pancreatic cancer cell proliferation is phosphatidylinositol 3-kinase dependent. J Surg Res 90: 39-44, 2000.

40. Zhang YX, Kong CZ, Wang HQ, Wang LH, Xu CL and Sun YH: Phosphorylation of Bcl-2 and activation of caspase-3 via the c-Jun N-terminal kinase pathway in ursolic acid-induced DU145 cells apoptosis. Biochimie 91: 1173-1179, 2009.

41. Kroemer G and Reed JC: Mitochondrial control of cell death. Nat Med 6: 513-519, 2000.

42. Ashkenazi A and Dixit VM: Death receptors: signaling and modulation. Science 281: 1305-1308, 1998.

43. Teraishi F, Zhang L, Guo W, et al: Activation of c-Jun NH2-terminal kinase is required for gemcitabine's cytotoxic effect in human lung cancer H1299 cells. FEBS Lett 579: 6681-6687, 2005.

44. Vivanco I and Sawyers CL: The phosphatidylinositol 3-kinase AKT pathway in human cancer. Nat Rev Cancer 2: 489-501, 2002 . 
45. Garcia-Echeverria C and Sellers WR: Drug discovery approaches targeting the PI3K/Akt pathway in cancer. Oncogene 27: 5511-5526, 2008.

46. Kane LP, Shapiro VS, Stokoe D and Weiss A: Induction of NF-kappaB by the Akt/PKB kinase. Curr Biol 9: 601-604, 1999.

47. Mayo LD and Donner DB: A phosphatidylinositol 3-kinase/Akt pathway promotes translocation of Mdm2 from the cytoplasm to the nucleus. Proc Natl Acad Sci USA 98: 11598-11603, 2001.

48. Zhou BP, Liao Y, Xia W, Zou Y, Spohn B and Hung MC: HER-2/ neu induces p53 ubiquitination via Akt-mediated MDM2 phosphorylation. Nat Cell Biol 3: 973-982, 2001.

49. Diehl JA, Cheng M, Roussel MF and Sherr CJ: Glycogen synthase kinase-3beta regulates cyclin D1 proteolysis and subcellular localization. Genes Dev 12: 3499-3511, 1998.
50. Pham NA, Tsao MS, Cao P and Hedley DW: Dissociation of gemcitabine sensitivity and protein kinase B signaling in pancreatic ductal adenocarcinoma models. Pancreas 35: E16-E26, 2007.

51. Ng SS, Tsao MS, Nicklee T and Hedley DW: Wortmannin inhibits pkb/akt phosphorylation and promotes gemcitabine antitumor activity in orthotopic human pancreatic cancer xenografts in immunodeficient mice. Clin Cancer Res 7: 3269-3275, 2001.

52. Sultana N: Clinically useful anticancer, antitumor, and antiwrinkle agent, ursolic acid and related derivatives as medicinally important natural product. J Enzyme Inhib Med Chem 26: 616-642, 2011. 